\title{
Abstract: AngioUnet \\ A Convolutional Neural Network for Vessel Segmentation in Cerebral DSA Series
}

\author{
Christian Neumann ${ }^{1}$, Klaus-D. Tönnies ${ }^{2}$, Regina Pohle-Fröhlich ${ }^{1}$ \\ ${ }^{1}$ Institut für Mustererkennung, Hochschule Niederrhein \\ ${ }^{2}$ Institut für Simulation und Graphik, Universität von Magdeburg \\ christian.neumann@hs-niederrhein.de
}

Das U-net [1] ist eine vielversprechende Architektur für Segmentierungsprobleme im Bereich der Medizin. Wir zeigen, wie sich diese Architektur effektiv auf die Segmentierung von zerebralen DSA Zeitserien anwenden lässt. Durch die Erweiterung der Eingabe auf mehrere Bilder wird es möglich besser zwischen Gefäsen und Hintergrund zu unterscheiden. Die Bilder wurden so gewählt, dass sie vier vordefinierte Ausbreitungszustände des Kontrastmittels zeigen. Da Arterien und Venen nicht direkt sondern über Kapillaren verbunden sind, lassen sich so Arterien von Venen unterscheiden. Desweiteren kann das U-net auf einem kleinen Korpus trainiert werden, wenn dies mit hilfreichen Data Augmentations wie Spiegelung, Rotation und Biasing kombiniert wird. Wir verwenden alle Rotationen um $90^{\circ}$, deren Spiegelungen und jeweils einen Bias von $\pm 5 \%$ des Dynamikumfangs wodurch insgesamt 24 Varianten pro Kachel entstehen. Unser Netzwerk erreicht einen Dice-Koeffizienten von $87.98 \%$ bei dieser Segmentierungsaufgabe. Wir vergleichen dies mit unterschiedlichen Konfigurationen und diskutieren den Einfluss von verschiedenen Artefakten wie sichtbaren Knochen, Kleber aus vorigen Embolisationen und die Schrauben und Marker des stereotaktischen Rahmens. Das vollständige Paper wird in den Proceedings der VISAPP 2018 erschienen sein [2].

\section{Literaturverzeichnis}

1. Ronneberger O, Fischer P, Brox T. U-Net: convolutional networks for biomedical image segmentation. arXiv preprint arXiv:150504597. 2015.

2. Neumann C, Tönnies KD, Pohle-Fröhlich R. AngioUnet: a convolutional neural network for vessel segmentation in cerebral DSA series. Proc VISIGRAPP. 2018. 